\title{
Modélisation spatiale et évaluation multicritère dans la détermination des sites propices à la production du maïs à Ouèssè, Bénin
}

\author{
Olatondji Salomon CHABI ADIMI ${ }^{1}$, Coovi Aimé Bernadin TOHOZIN ${ }^{2 *}$ et \\ Joseph OLOUKOI ${ }^{2}$ \\ ${ }^{I}$ Ecole Doctorale Pluridisciplinaire de l'UAC (Université d'Abomey-Calavi), \\ Laboratoire de Biogéographie et d'Expertise Environnementale, Bénin. \\ ${ }^{2}$ Institut Régional Africain des Sciences et Technologies de l'Information Géospatiale) \\ à OAU (Obafemi Awolowo University), Ilé-Ifè, Nigéria. \\ *Auteur correspondant,E-mail :tohozin2003@yahoo.fr; tohozin@rectas.org
}

\section{RESUME}

Au Bénin, le maïs occupe une place prépondérante dans le tissu productif. Il constitue le principal aliment de base de toute la partie méridionale du pays soit les $2 / 3$ de la population nationale. Malgré cette importance, son développement rencontre d'énormes difficultés. La présente étude visait à contribuer à une amélioration de la production du maïs dans la commune de Ouessè au Bénin. La méthodologie utilisée dans le cadre de ce travail est basée sur la télédétection et le Système d'Information Géographique couplé avec les méthodes d'analyse multicritère utilisant les données Landsat OLI-TIRS, ASTER-DEM, les couches hydrographiques, pédologiques et les composantes physico-chimique du sol. Au total, et de façon respective, l'étude révèle 43420 ha et 209928 ha de zones favorables et très favorables à la production du maïs contre 125 ha de zones peu favorables et 39625 ha qui sont défavorables. Le couplage du SIG avec les méthodes d'analyse multicritère facilite les bonnes prises de décision à caractère spatiale.

(C) 2018 International Formulae Group. All rights reserved.

Mots clés : Maïs, analyse multicritère, standardisation, Ouèsse, Bénin.

\section{ABSTRACT}

In Benin, maize occupies a prominent place in the productive fabric. It is the main staple food of the whole southern part of the country, i.e. $2 / 3$ of the national population. Despite this importance, its development encounters enormous difficulties. The present study aimed at contributing to an improvement of maize production in the commune of Ouessè in Benin. The methodology used in this work was based on remote sensing and Geographic Information System coupled with multi-criteria analysis methods using Landsat OLITIRS, ASTER-DEM data, hydrographic and pedologic layers, and soil physico-chemical components. In total, and respectively, the study reveals 43420 ha and 209928 ha of areas favorable and very favorable to the production of corn against 125 ha of less favorable areas and 39625 ha which are unfavorable. Coupling GIS with multicriteria analysis methods facilitates good spatial decision-making.

(C) 2018 International Formulae Group. All rights reserved.

Keywords: Maize, multicriteria analysis, standardization, Ouèsse, Benin. 


\section{INTRODUCTION}

L'augmentation croissante de la population constitue un défi pour les pays en voie de développement. Ils doivent avoir recours à une agriculture de qualité afin de satisfaire les besoins nutritionnels de cette population (Konan-waidhet et al., 2013).

Au cours de ces quarante dernières années, la performance économique de la plupart des pays d'Afrique subsaharienne a été sensiblement plus faible que celle des autres pays tropicaux en voie de développement. Cette contre-performance est liée à une mauvaise politique commune qui se caractérise par un manque de ressource favorable à l'agriculture et à la prévalence de certaines maladies Voortman (2003).

L'Afrique est la seule région du monde en développement où la production alimentaire moyenne par habitant a baissé au cours des 40 dernières années (FAO, 2001). Elle regroupe aujourd'hui le plus grand nombre de pays pauvres très endettés dans lesquels les populations souffrent de faibles revenus et de pénuries alimentaires. Pour une grande partie du secteur agricole africain, la croissance de la productivité et du commerce a été lente, et souvent négative (NEPAD, 2005).

Depuis 2006, l'économie béninoise est entrée dans une nouvelle phase de croissance. En effet, de 3,8\% en 2006, le taux de croissance est ressorti en 2008 à $5 \%$ en lien avec le rétablissement de la confiance au niveau des opérateurs économiques, la dynamisation de la production agricole. Cette expansion de l'économie ne s'est pas poursuivie en 2009 (MAEP, 2011). Il reste cependant un des pays les plus pauvres au monde. Une analyse des performances par secteur d'activité montre que le secteur primaire agricole a enregistré, sur la période 2007-2009, un taux de croissance moyen de $3,9 \%$ avec une contribution à la croissance économique estimée à $1,5 \%$. Ces performances sont en lien avec les niveaux de croissance enregistrés dans les trois soussecteurs dont l'agriculture avec 4,1\% (MAEP, 2011).

$\mathrm{Au}$ Bénin, la production du maïs représente $80 \%$ de la production céréalière (Soulé et al., 2004). Plusieurs études ont d'ailleurs montré l'importance de cette céréale
(Ofori et Kyei-Baffour, 2008 ; Nzossié et al., 2010 ; Adechian, 2015 ; Linda et al., 2015). Mais malheureusement, elle rencontre d'énormes difficultés avec la baisse de la fertilité des sols, les contraintes climatiques, etc. (Baco et al., 2011). Pour garantir une croissance pérenne de cette filière, il est important de repenser les différentes solutions susceptibles d'améliorer la productivité.

$\mathrm{Au}$ cœur de cette problématique se pose problème fondamental de la spatialisation pour une meilleure rentabilisation de cette filière : les endroits qui pourraient être consacrés à la culture pour une amélioration de la production. En général, l'analyse de la pertinence de tels outils vise à identifier le modèle spatial le plus approprié pour l'utilisation future des terres en fonction des exigences particulières, les préférences ou les prévisions d'une activité particulière (Collins et al., 2001). Ainsi, le Système d'Information Géographique (SIG) et l'évaluation multicritère (EMC) s'avèrent particulièrement utiles pour l'identification des sites propices à ce type d'aménagement (Kèdowidé, 2011).

Ainsi, la question est de savoir si les sites propices à la culture du maïs existent dans la commune de Ouessè. L'hypothèse qui en découle est que la commune de Ouessè dispose de sites spécifiques à la culture du maïs. L'objectif de la présente étude, au regard du sujet, des constats faits, de la question posée et de l'hypothèse formulée est d'identifier les sites potentiellement propices et spécifiques à la culture du maïs en vue d'améliorer les rendements. Spécifiquement, il s'agit de cartographier les facteurs déterminants dans l'identification des zones propices à la production du maïs et de déterminer ces zones. Ce travail illustre la méthodologie et les résultats obtenus par une modélisation géomatique conduisant à l'identification des sols favorables à la production du maïs. Le présent travail est structuré en quatre points : la présentation de la zone d'étude ; le matériel et les méthodes ; puis les résultats suivis de discussion.

\section{MATERIEL ET METHODES}

Présentation de la zone d'étude

Située entre $8^{\circ} 7^{\prime} 56^{\prime \prime}$ et $8^{\circ} 46^{\prime} 7^{\prime \prime}$ de latitude Nord et entre $2^{\circ} 11^{\prime} 22^{\prime \prime}$ et $2^{\circ} 45^{\prime} 57^{\prime \prime}$ 
de longitude Est, la commune de Ouèssè se localise entre les fleuves Okpara à l'Est et Ouémé à l'Ouest et une superficie d'environ $3000 \mathrm{~km}^{2}$, soit $2,56 \%$ de la superficie nationale (Figure 1).

De par sa position géographique, la Commune de Ouèssè est située dans la cinquième zone agro-écologique et jouit d'un climat soudano-guinéenne. Le régime pluviométrique est à cheval sur celui de la distribution bimodale du Sud et celui de la distribution uni modale du Nord et est caractérisé par deux saisons (Oloukoi, 2012). Les sols ferrugineux concrétionnés sont les plus dominants. On y rencontre également des sols ferrugineux et des sols ferralitiques faiblement désaturés à faible échelle et des sols hydromorphes développés le long des cours d'eaux. Elle fait partie des communes les plus arrosées avec environ $292 \mathrm{~km} \mathrm{de}$ cours d'eau.

\section{Méthodes}

Les données utilisées dans le cadre de cette étude constituent la base des critères. Il s'agit :

- de l'image satellite Landsat OLI-TIRS, de 2014 de résolution $30 \mathrm{~m}$; qui a permis de générer le critère d'occupation du sol qui constitue la seule contrainte de ce modèle ;

- de l'image satellite ASTER-DEM, de résolution $30 \mathrm{~m}$ de 2000 a permis d'obtenir le facteur topographique ;

- des couches pédologiques et hydrographiques qui ont permis d'établir respectivement le facteur d'aptitude pédologique de chaque sol et l'accessibilité au cours d'eau ;

- du facteur relatif aux composantes physico-chimiques du sol à savoir: le Potentiel d'Hydrogène $(\mathrm{pH})$, le Phosphore $(\mathrm{P}), \quad$ l'azote $(\mathrm{N})$ et le potassium $(\mathrm{K})$ obtenues à partir d'échantillon de sol. Le prélèvement s'est fait entre la surface et une profondeur de $15 \mathrm{~cm}(0$ à $15 \mathrm{~cm})$.

$\mathrm{Au}$ total, un prélèvement de 48 échantillons s'est fait en tenant compte d'un quadrillage de $10 \mathrm{~km} \times 10 \mathrm{~km}$ (Rilwani et Ikhuoria, 2011). Les coordonnées géographiques des différents échantillons du sol ont été prises (Figure 2).

\section{Traitement des données}

La méthode multicritère utilisée est basée sur la pondération des critères à partir de la méthode PHA (Processus de Hiérarchisation Analytique), qui permet d'attribuer des poids aux différents critères en fonction de leur importance. La pondération des critères est précédée par leur standardisation avant d'être agrégés pour la cartographie des zones propices à la production du maïs. Cette méthode exige un traitement bien précis des données qui constituent la base des critères. Dans cette perspective,

- l'image Landsat OLI-TIRS a fait l'objet d'une classification supervisée pour l'obtention de l'occupation du sol ;

- l'image ASTER-DEM a permis de générer la pente en utilisant le logiciel ArcGIS ;

- la couche pédologique a été rastérisée pour dresser le critère d'aptitude pédologique ;

- les couches hydrographiques ont été intégrées dans ArcGIS pour générer la distance de coût d'accès aux sources d'eau ;

- la teneur en $\mathrm{pH}$, en phosphore en potassium et en azote du sol a été obtenue à partir de la méthode d'interpolation en utilisant la technique du Kriging.

\section{Standardisation des critères}

$\mathrm{La}$ mise en place des cartes critères exige une standardisation au préalable; ce qui permet de les quantifier en fonction de leurs aptitudes. Elle est basée sur une reclassification continue (Jiang et Eastman, 2010).

\section{Tableau de pondération}

Le tableau de pondération a été dressé sur la base des études déjà réalisées et des travaux de terrain et a permis de pondérer chaque critère. La méthode PHA de Saaty (1980) a été utilisée. Elle consiste à comparer les critères deux à deux en termes d'importance relative par rapport à l'objectif défini sur la base d'une échelle de pondération. La pondération des critères est prépondérante et influe directement sur la qualité du choix effectué (Tableau 1).

Le Tableau 1 se base sur un principe selon lequel si un critère $A$ est 3 fois plus important qu'un critère $B$ alors $B$ est $1 / 3$ de 
fois important que A. De plus, si le critère $\mathrm{A}$ est plus important que le critère $\mathrm{B}$ et $\mathrm{C}$ plus important que $\mathrm{A}$ alors C'est plus important que B. C'est sur la base de cette logique que le tableau de pondération a été mise en place.

Afin de tester la cohérence de la réponse qui indique si les données ont un rapport logique entre elles, Saaty (2008), propose de suivre le procédé suivant les formules des équations 1,2 et 3 :

Valeur calculée sur la base d'indice de Saaty

$\lambda \max =\sum\left(\sum c i . w i\right)(1)$

L'indice de cohérence (IC):

IC $=\frac{\lambda \max -n}{n-1}$

Le ratio de cohérence (RC) :

$R C=\frac{I C}{I A}$

$\mathrm{C}=$ colonne, $\mathrm{n}$ le nombre de facteur et $\lambda$ max, une valeur calculée sur la base de la matrice de Saaty, IC l'Indice de Cohérence.

Si RC> $10 \%$, il n'y a pas de cohérence dans la comparaison des critères par paire. $\mathrm{La}$ matrice doit être réévaluée. IA est un indice aléatoire fixé en fonction du nombre de critère.

Les indices aléatoires en fonction du nombre de critère ont été calculé (Tableau 2 ).

$$
\begin{aligned}
& \lambda \max =(2,6 \times 0,365)+(7 \times 0,155)+ \\
& (7 \times 0,155)+(7 \times 0,155)+(0,056 \times 17)+ \\
& (0,056 \times 17)+(0,056 \times 17)
\end{aligned}
$$$$
=0,949+3,276+2,856
$$

$\lambda \max =7,081$

$\mathrm{IC}=(7,081-7) / 6=0.0135$

$\mathrm{RC}=0,01 / 1,32=0,01 \leftrightarrow \mathrm{RC}=1 \%$

Or selon Saaty si RC > 10\% alors il n'existe pas de cohérence dans la comparaison par paire.

Or dans le cas de cette étude $\mathrm{RC}=1 \%<10$

Le ratio de cohérence étant inférieur à $10 \%$ alors la cohérence est acceptable.

\section{Agrégation}

Une fois les unités partielles mises en place et pondérées, elles vont faire l'objet d'une agrégation par combinaison linéaire pondérée sur la base de la formule de l'équation 4 en utilisant le logiciel ArcGIS.

$$
\sum_{i} w i x i \times \prod_{j} c j
$$

Source : Estoque (2011)

Avec $\mathrm{x}$ le facteur, w le poids et $\mathrm{c}$ la contrainte

Signalons que les échantillons du sol prélevés dans la zone d'étude ont fait l'objet d'une analyse potentiométrique au laboratoire d'analyse d'eau de la Direction Départementale de l'Energie et de l'Eau (Borgou-Alibori) pour extraire les valeurs de $\mathrm{pH}$, potassium, phosphore, azote de chaque échantillon. Ces valeurs ont été interpolées à l'aide de la technique de kringing dans le logiciel ArcGIS 10.1.

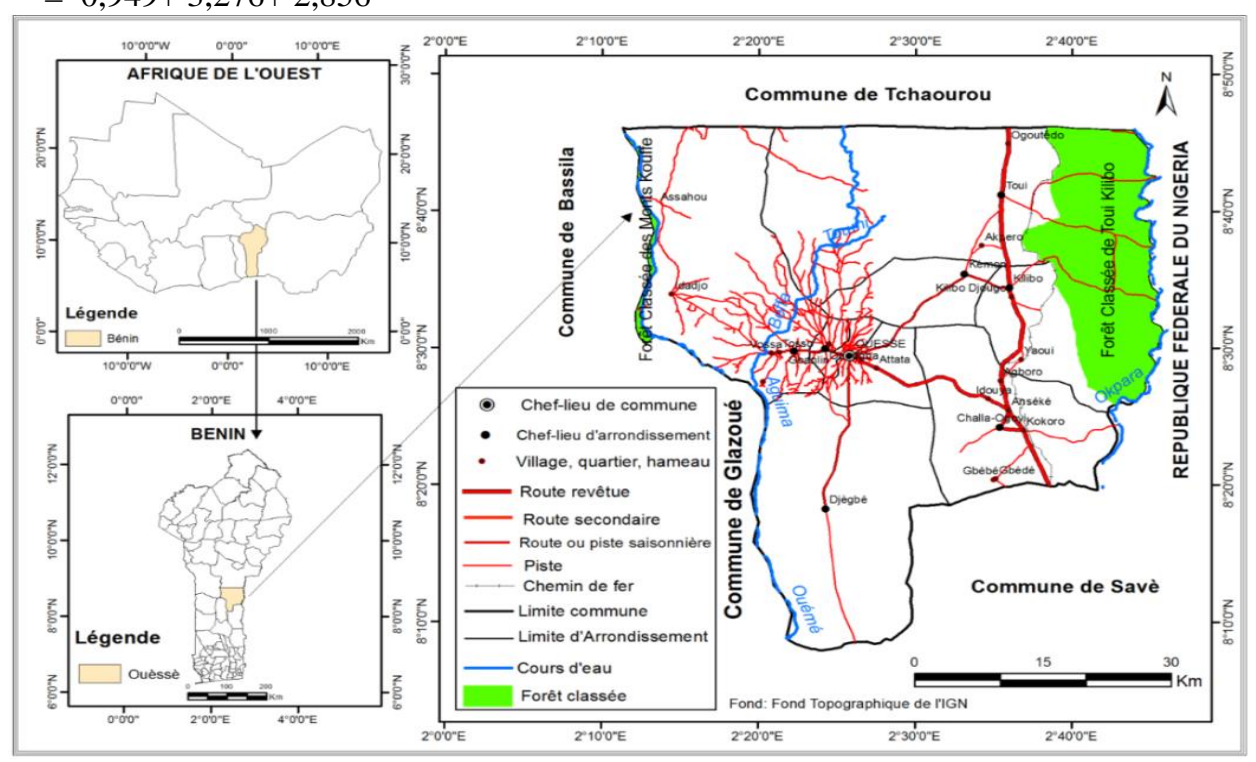

Figure 1 : Situation géographique de la commune de Ouessè. 


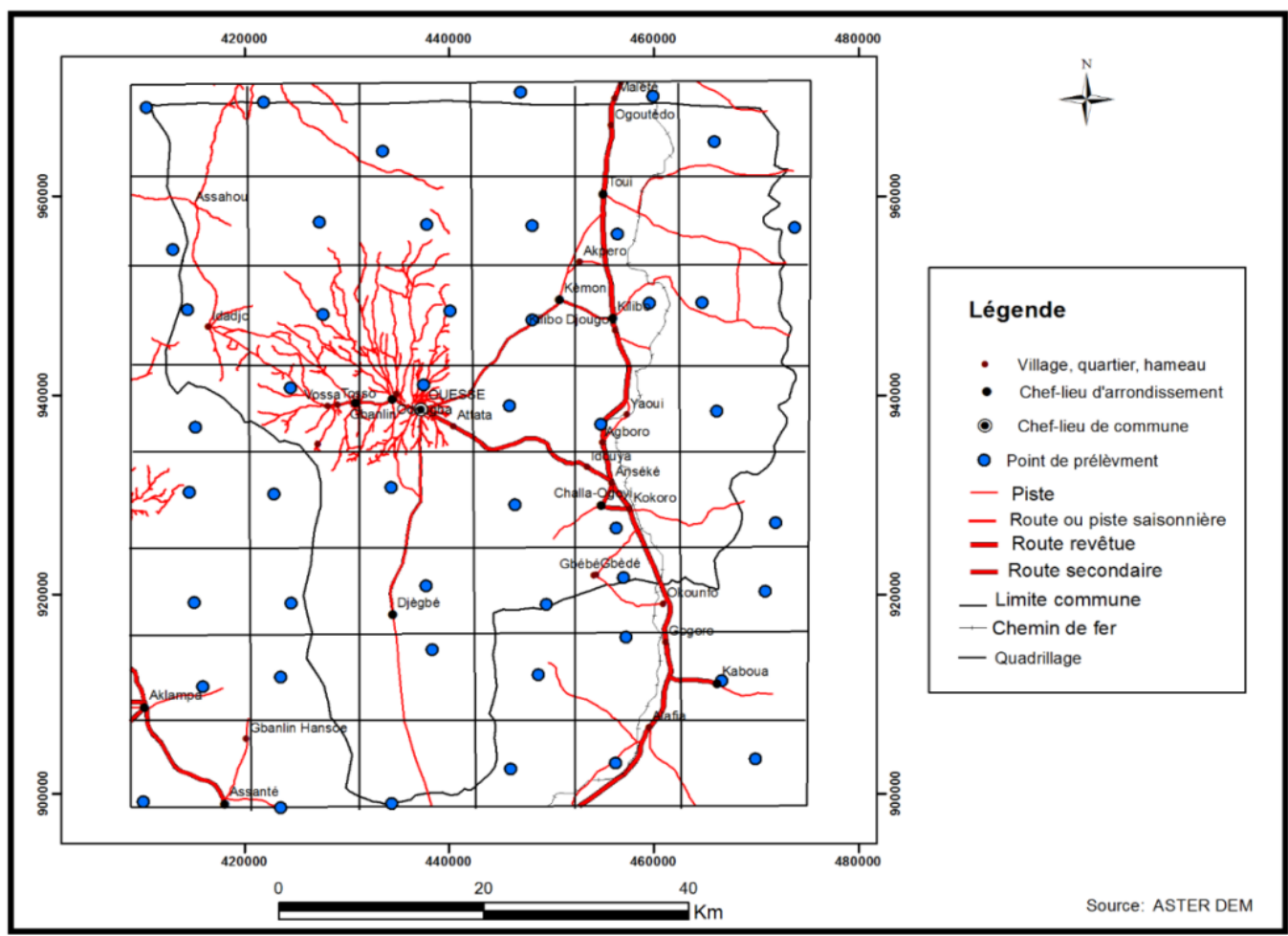

Figure 2 : Distribution spatiale des points de prélèvement d'échantillons du sol.

Tableau 1: Echelle de pondération de Saaty.

\section{Comparaison d'un critère par rapport à un autre}

Intensité de l'importance

Même importance que

Modérément plus important que

Fortement plus important que

Très fortement plus important que

Extrêmement plus important que

Modérément moins important que

Fortement moins important que

Très fortement moins important que

Extrêmement moins important que 
Tableau 2: Indice aléatoire.

\begin{tabular}{lllllllllll}
\hline Nombre de critères & $\mathbf{2}$ & $\mathbf{3}$ & $\mathbf{4}$ & $\mathbf{5}$ & $\mathbf{6}$ & $\mathbf{7}$ & $\mathbf{8}$ & $\mathbf{9}$ & $\mathbf{1 0}$ & $\mathbf{1 1}$ \\
\hline IA & 0 & 0,58 & 0,90 & 1,12 & 1,24 & 1,32 & 1,41 & 1,45 & 1,49 & 1,51 \\
\hline Saaty, 1980 & & & & & & & & & &
\end{tabular}

\section{RESULTATS}

Les résultats se déclinent en deux parties essentielles: l'identification des critères et l'agrégation pour l'identification des zones propices à la culture du maïs.

L'identification des zones propices à la culture du maïs dans la commune de Ouessè est basée sur plusieurs critères. Les critères pédologiques: l'aptitude pédologique des types de sol et contenance en élément physico-chimique tels que l'azote, le $\mathrm{pH}$, le phosphore et le potassium, l'accessibilité aux sources d'eau et le critère d'occupation du sol. La Figure 3 présente en (a) le $\mathrm{pH}$ du sol, (b) le facteur relatif au $\mathrm{pH}$, (c) le phosphate du sol et (d) le facteur relatif au phosphate. L'analyse de ces résultats permet de constater que les sols ayant un $\mathrm{pH}$ supérieur à 6,1 sont les plus aptes à la culture du maïs et ont un indice d'aptitude maximal qui est 10 . Plus la valeur du $\mathrm{pH}$ décroit, plus l'indice d'aptitude décroit. Le phosphate est présent en grande quantité dans le Sud et le Nord du secteur d'étude.

Ensuite, la Figure 4 montre en (e) le potassium du sol, (f) le facteur relatif au potassium, (g) l'azote du sol et (h) le critère d'occupation du sol. La Figure 5 présente en (i) le facteur pédologique et (j) le facteur d'accessibilité à l'eau.

Ces figures présentent les différents critères qui influencent le modèle de l'identification des sites propices à la culture du maïs dans la commune de Ouessè. Le facteur relatif à l'azote du sol n'a pas été représenté car il ressort de l'analyse de la Figure 4 (g) que l'azote n'est pas un facteur limitant à Ouessè. La teneur en azote varie entre 0,614167 et $0,846667 \mathrm{mg} / \mathrm{Kg}$. Alors que lorsque la teneur en azote atteint un seuil de $0,26 \mathrm{mg} / \mathrm{Kg}$, la zone présente $100 \%$ d'aptitude à la production du maïs.

\section{Pondération des critères}

Dans un problème de décision multicritère, le décideur considère souvent que les facteurs n'ont pas la même importance. Il faut cependant qu'un poids soit donc attribué à chacun d'eux. La pondération des critères constitue donc la base de toutes opérations multicritères car elle influence de façon directe les résultats. Sur la base de ces différentes explications, la comparaison par paire de chaque critère a été élaborée (Tableau $3)$.

Ce tableau a été mis en place à partir des exigences agronomiques du maïs et de l'échelle de pondération de Saaty. Ainsi, à partir du tableau de comparaison, la pondération de chaque critère a été établie (Tableau 4).

De l'analyse du Tableau 4, il ressort que la pédologie est le facteur qui détient le poids le plus élevé dans la détermination des zones propices à la culture du maïs. Elle est suivie de la proximité des sources d'eau de la teneur en $\mathrm{pH}$ et en phosphore du sol et enfin les dernières gammes les moins importantes sont la teneur en potassium, en azote du sol et la topographie.

\section{Agrégation et identification des zones propices à la culture du maïs}

L'aide à la décision vise très souvent à élaborer des recommandations sur lesquelles le décideur s'appuiera pour définir un plan d'action. En analyse multicritère, cette recommandation se base sur l'agrégation qui est l'étape la plus importante et permet de synthétiser les différents critères intervenant dans ce processus de décision après leur pondération. Il existe plusieurs approches d'agrégation en analyse multicritère. Les variables sont des plans d'information et les 
opérateurs sont ceux de l'analyse et la modélisation spatiale (Figure 6).

La commune de Ouessè présente une aptitude élevée à la production du maïs. Les zones favorables et très favorables sont pour la plupart proches des sources d'eaux et peuvent bénéficier d'un aménagement hydroagricole orienté vers les techniques de maîtrise de l'eau pendant la saison sèche ou pendant la période de bouleversement de la pluviométrie.
Elles totalisent respectivement une superficie de 43420 ha et 209928 ha soit environ $15 \%$ et $71,5 \%$ de la superficie de la commune de Ouessè. Quant aux zones impossibles, ce sont les zones où toute production du maïs reste impossible. Elles concernent les espaces bâtis, les forêts classées et l'eau. Elles totalisent une superficie de 39625 ha soit environ $13 \%$ de la superficie de la commune contre 125 ha pour les zones peu favorable soit environ $0,04 \%$.

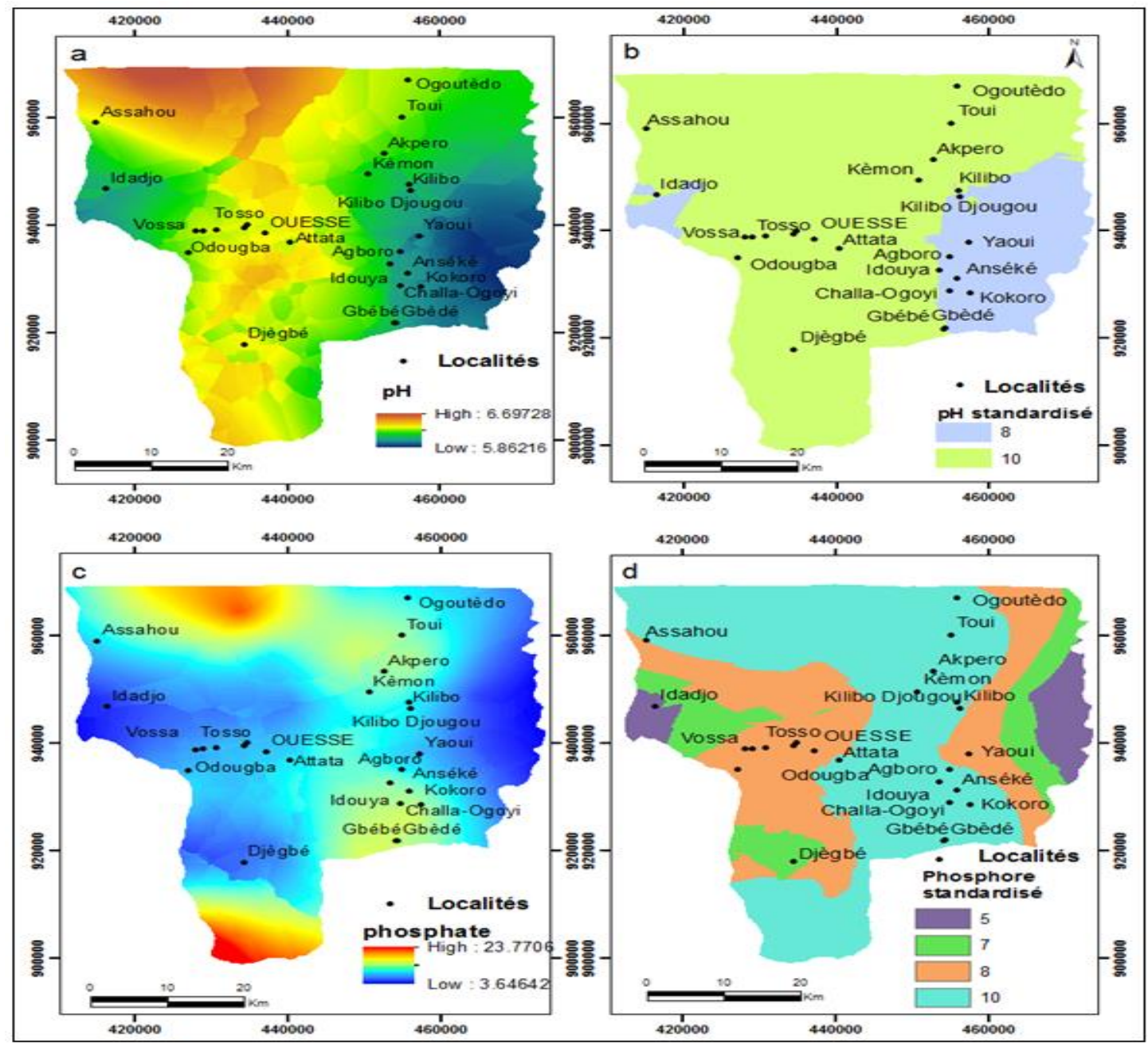

Figure 3 : Représentation du pH et du phosphate. (a) pH du sol, (b) Facteur relatif au pH, (c) Phospha te du sol et (d) Facteur relatif au phosphate. 


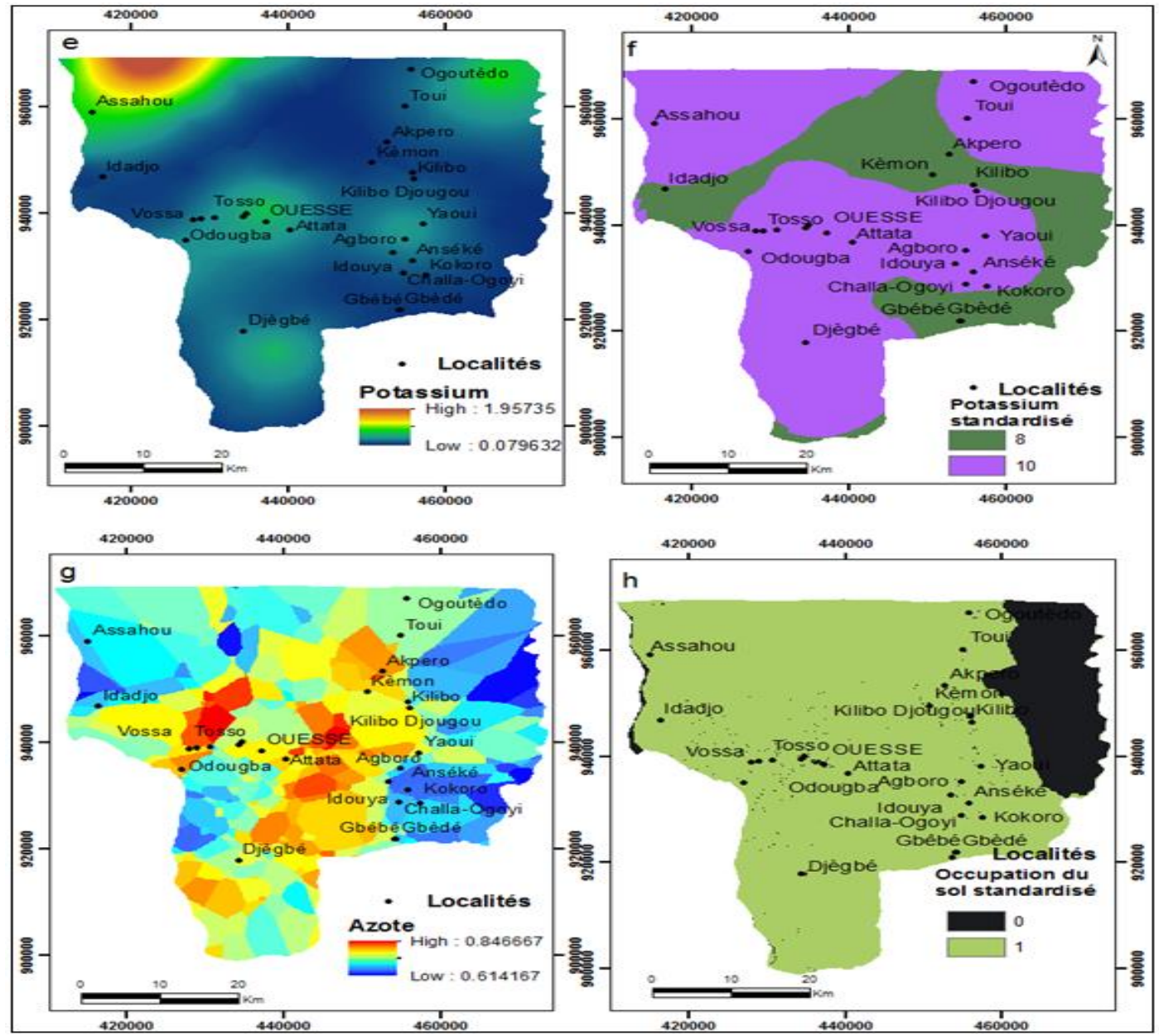

Figure 4 : Représentation du potassium, de l'azote et de l'occupation du sol. (e) Potassium du sol, (f) Facteur relatif au potassium, (g) Azote du sol et (h) Critère d'occupation du sol.

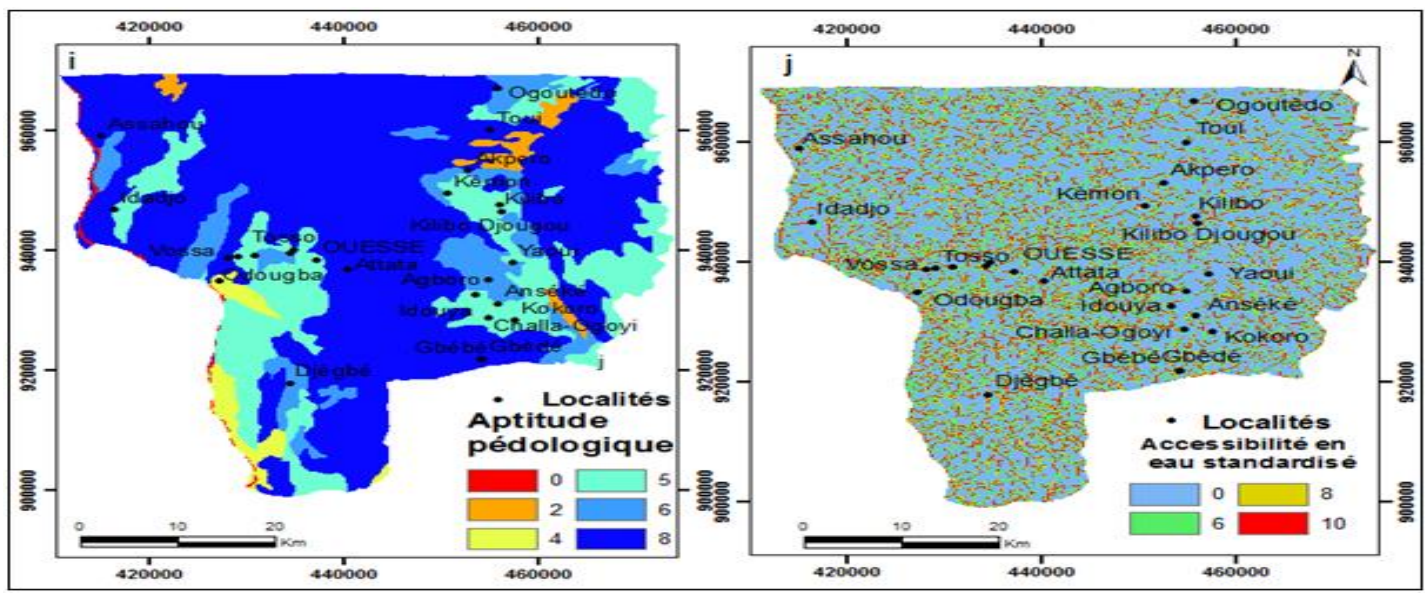

Figure 5 : Représentation de l'aptitude pédologique et de l'accessibilité en eau. (i) Facteur pédologique et (j) Facteur d'accessibilité à l'eau. 
Tableau 3: Comparaison par paire des facteurs.

\begin{tabular}{lccccccc}
\hline Critères & Pédologie & $\begin{array}{c}\text { Proximité de } \\
\text { cours d'eau }\end{array}$ & $\mathbf{p H}$ & Phosphore & Potassium & Azote & Pente \\
\hline Pédologie & 1 & 3 & 3 & 3 & 5 & 5 & 5 \\
Proximité cours d'eau & $1 / 3$ & 1 & 1 & 1 & 3 & 3 & 3 \\
$\mathrm{pH}$ & $1 / 3$ & 1 & 1 & 1 & 3 & 3 & 3 \\
Phosphore & $1 / 3$ & 1 & 1 & 1 & 3 & 3 & 3 \\
Potassium & $1 / 5$ & $1 / 3$ & $1 / 3$ & $1 / 3$ & 1 & 1 & 1 \\
Azote & $1 / 5$ & $1 / 3$ & $1 / 3$ & $1 / 3$ & 1 & 1 & 1 \\
Pente & $1 / 5$ & $1 / 3$ & $1 / 3$ & $1 / 3$ & 1 & 1 & 1 \\
Total & 2.6 & 7 & 7 & 7 & 17 & 17 & 17 \\
\hline
\end{tabular}

Tableau 4 : Tableau de pondération.

\begin{tabular}{lllllllll}
\hline Critères & Pédologie & Cours d'eau & pH & Phosphore & Potassium & Azote & Pente & Poids \\
\hline Pédologie & 0,384 & 0,428 & 0,428 & 0,428 & 0,294 & 0,294 & 0,294 & 0,365 \\
Cours d'eau & 0,128 & 0,142 & 0,142 & 0,142 & 0,176 & 0,176 & 0,176 & 0,155 \\
pH & 0,128 & 0,142 & 0,142 & 0,142 & 0,176 & 0,176 & 0,176 & 0,155 \\
Phosphore & 0,128 & 0,142 & 0,142 & 0,142 & 0,176 & 0,176 & 0,176 & 0,155 \\
Potassium & 0,076 & 0,047 & 0,047 & 0,047 & 0,058 & 0,058 & 0,058 & 0,056 \\
Azote & 0,076 & 0,047 & 0,047 & 0.047 & 0,058 & 0,058 & 0,058 & 0,056 \\
Pente & 0,076 & 0,047 & 0,047 & 0,047 & 0,058 & 0,058 & 0,058 & 0,056 \\
Total & 1 & 1 & 1 & 1 & 1 & 1 & 1 & 1 \\
\hline
\end{tabular}

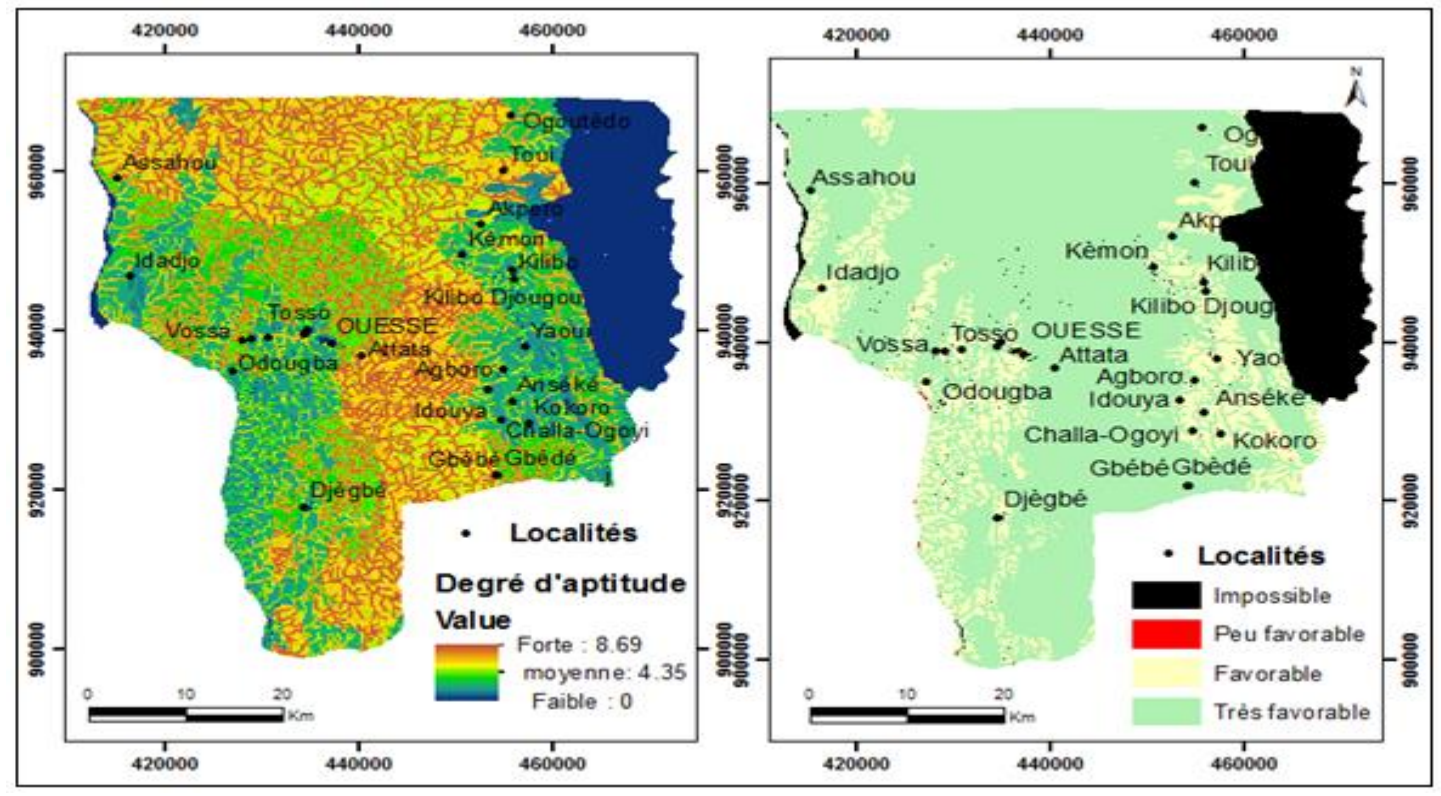

Figure 6 : Aptitude à la production du maïs dans la commune de Ouessè. 


\section{DISCUSSION}

La confrontation des résultats avec ceux de travaux antérieurs permet de bien les appréhender en vue de la validation scientifique et de l'opérationnalisation des conclusions. Trois éléments importants sont à retenir : l'importance de l'utilisation de l'analyse multicritère; l'efficacité du couplage SIG et AMC et les difficultés et limites de cette étude.

\section{Importance de l'utilisation de l'analyse multicritère}

L'analyse multicritère repose sur un ensemble de procédures permettant de détailler un problème décisionnel portant sur des situations complexes. Dans l'analyse multicritère, on cherche un domaine de résolution pouvant tenir compte de l'ensemble des critères susceptibles d'influencer la décision. Le critère se définit comme un facteur à prendre en considération pour évaluer un scénario donné ou pour apprécier une occasion d'action (Prévil et al., 2003). D'autres auteurs ( Ben Mena, 2000 ; Martin et al., 2007 ; Diaby et al., 2010) ont montré leur efficacité sur le terrain.

Les principes de l'analyse multicritère à la décision (AMCD), témoignent autant d'une évolution des pratiques des utilisateurs que d'un changement profond dans les modalités du processus décisionnel (Prévil et al., 2003). L'AMCD permet de composer avec la multiplicité, la divergence et la nature (quantitative ou qualitative) des critères en vue d'aboutir à des compromis acceptables (Simos cité par Prévil et al., 2003 ).

\section{Efficacité du couplage SIG et AMC}

La présente recherche a montré l'importance du SIG et de la méthode d'analyse multicritère dans la détermination des sites propices à la production du maïs. Ce couplage a favorisé considérablement cette étude et a permis d'obtenir de bons résultats. Des résultats similaires ont été obtenus par Kêdowidé (2010). Pour cet auteur, les méthodes d'AMC toutes seules sont incapables de tenir compte de tous les aspects des problèmes de décision à référence spatiale. Le SIG est bien adapté pour répondre à cette insuffisance de l'approche classique de l'analyse multicritère spatiale. L'intégration du SIG et l'AMC semble donc être la meilleure solution pour combler leurs lacunes respectives. Ouédraogo et al. (2012), ont confirmé les résultats extraordinaires qui peuvent subvenir de ce couplage. En effet, selon ces auteurs, dans le renforcement du SIG comme un outil d'aide à la décision à référence spatiale, une solution possible consiste en son intégration à l'analyse multicritère (AMC).

L'efficacité de cette combinaison a été démontrée par des auteurs comme (SaintLaurent et Schneider, 2004 ; Paegelow et al., 2004 ; Malczewski, 2006; Chakhar, 2006, Kêdowidé, 2010 ; Maddahi et al., 2014 ; Chabi Adimi, 2015). Pour ces différents auteurs, cette combinaison de l'analyse multicritère aux systèmes d'information géographique, permet de répondre à des problèmes de localisation, d'analyse de contraintes, de potentiels ou de risques. Elle constitue une voie privilégiée et incontournable pour faire évoluer les SIG vers de véritables systèmes d'aide à la décision.

Le présent article a montré l'importance des SIG et des méthodes d'analyses multicritères dans la détermination des zones propices à la production du maïs. Les résultats obtenus corroborent de façon générale les nombreuses études menées dans le domaine des SIG couplés avec les analyses multicritères. C'est notamment celles réalisées par Jiang et Eastman (2000), Kouamé et al. (2007), (Kêdowidé (2011), Estoque (2011) et Konan-Waidhet et al. (2013).

Les différents critères exploités en ce qui concerne cette étude ont de façon réelle permis d'identifier des sites propices à la production de maïs dans ce milieu. Les procédures d'analyse spatiale basée sur des critères pour l'identification et le choix de l'emplacement d'un site a déjà fait ses preuves à travers diverses études (Bensaid et al., 2007 ; Kêdowidé, 2010 ; Dembélé et al., 2014). 


\section{Conclusion}

Le SIG couplé avec les méthodes d'analyse multicritère offrent de grandes possibilités dans l'aide à la décision à caractère spatiale. Ils intègrent tous les paramètres relatifs à ces types d'aménagement. Les résultats issus de l'application de ces méthodes ont permis d'identifier les zones propices à la culture du maïs dans cette zone. En effet, Ouessè présente dans l'ensemble un degré d'aptitude élevé à la production du maïs. Bien que la commune présente un degré d'aptitude élevé pour cette production, le rendement reste faible et est en moyenne d'une tonne à l'hectare. Cette faiblesse résulte de nombreuses contraintes qui entravent son développement. Ces contraintes sont entre autres, la variabilité climatique, les problèmes liés à la transhumance, le manque ou la précarité d'accès aux systèmes financiers.

\section{CONFLIT D'INTERETS}

La réalisation de ce travail n'a suscité aucun conflit d'intérêts de quelque nature que ce soit pouvant entacher sa crédibilité.

\section{REMERCIEMENTS}

Les auteurs remercient très sincèrement les autorités de la commune de Ouèssè pour leur franche collaboration.

\section{REFERENCES}

Adechian SA, Baco MN, Akponikpe I, Imorou Toko I, Egah J, Affoukou K. 2015. Les pratiques paysannes de gestion des pesticides sur le maïs et le coton dans le bassin cotonnier du Bénin, VertigO - La Revue Electronique en Sciences de l'Environnement, 15(2): DOI : 10.4000/vertigo.16534

Baco MN, Abdoulaye T, Sanogo D, Langyintuo A. 2011. Caractérisation des Ménages Producteurs de Maïs en Zone de Savane Sèche au Bénin. INRAB IITA : Cotonou ; 38.

Ben Mena S. 2000. Introduction aux méthodes multicritères d'aide à la décision.
Biotechnol. Agron. Soc. Environ., 4(2): 83-93.

Bensaid A, Barki M, Talbi O, Benhanifia K, Mendas A. 2007. L'analyse multicritère comme outil d'aide à la décision pour la localisation spatiale des zones à forte pression anthropique : le cas du département de Naâma en Algérie. Revue Télédétection, 7(1-2-3-4): 359371, https://www.researchgate.net/ publication/242250347

Chabi Adimi OS. 2015. SIG et identification des sites propices à la production du maïs dans la commune de Ouèssè au Bénin. Mémoire de Master au Campus universitaire Obafemi Awolowo, RECTAS, $121 \mathrm{p}$.

Chakhar S. 2006. Cartographie décisionnelle multicritère : Formalisation et implémentation informatique. Thèse de Doctorat de l'Université Dauphine, DFR Sciences des organisations, Université Paris, $288 \mathrm{p}$.

Debrincat L, Meyère A. 1998. L'aide multicritère à la décision : des potentialités pour l'évaluation des projets de transport collectif en Île-de-France. Metropolis, 106-107: 57-63.

Dembélé M, Toko Mouhamadou I, Tohozin CAB. 2014. Identification des sites favorables à la production de mangues dans le cercle de Bougouni au Mali. Revue de Géographie de l'Université de Ouagadougou, 003: 123-136.

Diaby M, Valognes F, Demange AC. 2010. Utilisation d'une méthode multicritère d'aide à la décision pour le choix des clones d'hévéa à planter en Afrique. Base, 14(2): 299-309.

Estoque RC. 2011. GIS-based Multi-Criteria Décision Analysis (in Natural Resource Management), Note de cours, Université de Tsukuba, $24 \mathrm{p}$.

FAO. 2001. Le rôle de l'agriculture dans le développement des pays les moins avancés et leur intégration à l'économique mondiale, http://www.fao.org/docrep 
Jiang H, Eastman JR. 2000. Application of fuzzy measures in multi-criteria evaluation in GIS. International Journal of Geographical Information Science, 2(14): 173-184.

Kêdowidé MGC. 2010. Modélisation géomatique par évaluation multicritère pour la prospection de sites d'agriculture à Ouagadougou. VertigO - La Revue en Sciences de l'Environnement, 10(2): 20. http://vertigo.revues.org/10368.

Kêdowidé MGC. 2011. SIG et analyse multicritère pour l'aide à la décision en agriculture urbaine dans les pays en développement, cas de Ouagadougou au Burkina Faso. Thèse de doctorat, Université Paris 8, $281 \mathrm{p}$.

Konan-Waidhet AB, Kouadio KE, Dibi B, Bakayoko S, Savane I, Lazar G. 2013. Apport d'un système d'information géographique et de l'évaluation multicritère dans la modélisation des sites propices à la riziculture dans le Nord-Ouest de la Côte d'Ivoire (Denguelé). Tunis, 18 p. http://www.geosp.net.

Kouamé KJ, Deh SK, Anani AT, Jourda JP, Biémi J. 2007. Gestion des déchets solides dans le District d'Abidjan (Sud de la Côte d'Ivoire) : Apports d'un SIG et des méthodes d'analyse multicritère. Actes de la conférence SIG 2007 (Versailles, France), Édit. ESRI, Versailles.

Linda A, Oluwatola A, Opeyemi TA. 2015. Land Suitability Analysis for Maize Production in Egbeda Local Government Area of Oyo State Using GIS Techniques. International Journal of Biological, Food, Veterinary and Agricultural Engineering, 9(3): 270-275.

Maddahi Z, Jalalian A, Zarkesh M. M. K, Honarjo N. 2014. Land suitability analysis for rice cultivation using multi criteria evaluation approach and GIS. European Journal of Experimental Biology, 4(3): 639-648.
MAEP. 2011. Plan Stratégique de Relance du Secteur Agricole (PSRSA). MAEP: Cotonou, 108.

Malczewski J. 2006. GIS-based multicriteria decision analysis: a survey of the literature. International Journal of Geographical Information Science, 7(20): 703-726. http://dx.doi.org/ 10.1080/13658810600661508

Martin C, Ruperd Y, Legret M. 2007. Urban storm water drainage management: the development of a multicriteria decision aid approach for best management practices. Eur. J. Oper. Res., 181(1): 338-349. https://doi.org/10.1016/ j.ejor.2006.06.019

NEPAD. 2005. Avantage Comparative des Produits Agricoles en Afrique. FAO: Rome; 100.

Nzossié EJF, Ndamè JP, Temple L, Dury S, Ndjouenkeu R, Kamdem MS. 2010. L'émergence du maïs dans la consommation alimentaire des ménages urbains au Nord-Cameroun. Économie Rurale, [En ligne], 318-319. URL : http://economierurale.revues.org/2769

Ofori E, Kyei-Baffour N. 2008. Agrometeorology and maize production: Chapter $13 \mathrm{C}$ of Guide to Agricultural Meteorological Practices (GAMP). WMO/CAgM, 1-19.

Oloukoi J. 2012. Utilisation de la télédétection et des systèmes d'information géographique dans l'étude de la dynamique spatiale de l'occupation des terres au centre du Bénin. Thèse de Doctorat, Université d'Abomey-Calavi, $304 \mathrm{p}$.

Paegelow M, Villa N, Cornez L, Ferraty F, Ferré L, Sarda P. 2004. Modélisations prospectives de l'occupation du sol. Le cas d'une montagne méditerranéenne. Cybergeo: European Journal of Geography, Systèmes, Modélisation, Géostatistiques, article 295, URL : http://cybergeo.revues.org/2811.

Prévil C, Thériault M, Rouffignat J. 2003. Analyse multicritère et SIG pour faciliter la concertation en aménagement du 
territoire : vers une amélioration du processus décisionnel ? Cahiers de Géographie du Québec, 47(130): 35-61. URI:

http://id.erudit.org/iderudit/007968ar, DOI: 10.7202/007968ar

Rilwani M, Ikhuoria I. 2011. Geoinformaticsbased land suitability assessment of a rain forest river bassin for precision crop production in Nigeria. Lagos Journal of Geo-Information Science, 1(1): 19-30.

Voortman RL. 2003. African Land Ecology: Opportunities and Constraints for Agricultural Development. Ambio, 5(32): 367-373. DOI: 10.1639/00447447(2003)032[0367:

ALEOAC] 2.0.CO; 2

Saaty TL. 1980. The Analytic Hierarchy Process, Planning, Priority Setting, Resource Allocation. McGraw-Hill: New York.
Saaty TL. 2008. Decision Making with the Analytic Hierarchy Process. International Journal of Services Sciences, 1: 83-98. DOI: 10.1504/IJSSCI.2008.017590

Saint-Laurent D, Schneider M. 2004. Application d'un système d'information géographique à l'analyse cartographique des berges sensibles à l'érosion (Rivière du Lièvre, Québec, Canada). Bulletin de la Société Géographique de Liège, 44: 71-86.

Schärlig A. 1985. Décider sur Plusieurs Critères: Panorama de l'Aide à la Décision. PPUR : Lausanne; 237.

Simos J. 1990. Évaluer l'Impact sur l'Environnement, une Approche Originale par l'Analyse Multicritère et la Négociation. Presses Polytechniques et Universitaires Romandes : Lausanne. 